\title{
STAR PRODUCT AND ITS APPLICATION TO THE MIC-KEPLER PROBLEM
}

\author{
TOMOYO KANAZAWA AND AKIRA YOSHIOKA
}

Communicated by Ivaïlo M. Mladenov

\begin{abstract}
We show that the MIC-Kepler probrem is simply solved via the phasespace formulation of non-relativistic quantum mechanics. The MIC-Kepler problem is the Hamiltonian system behind the hydrogen atom subjected to the influence of the Dirac's magnetic monopole field and the square inverse centrifugal potential force besides the Coulomb's potential force. We get the energy spectrum of the bound states explicitly and construct the Green's functions for $E<0$ by means of the Moyal product, which is one of the 'star' products denoted by ' $*$ '.
\end{abstract}

\section{Introduction}

In 1978, Bayen et al [1] demonstrated that the quantum mechanics could be replaced by a "deformation" of the classical mechanics by introducing an associative algebra (*-product algebra) and the corresponding Lie algebra. The Moyal product and the associated Moyal bracket are the most familiar instance of them, which are directly connected with the definition of quantum commutator.

In this paper, using a star product, more precisely using the Moyal product, we calculate the energy spectrum and Green's functions of the MIC-Kepler problem in the Weyl-Wigner-Moyal (WWM) formalism, which furnishes an alternative formulation - historically, the latest - of quantum mechanics that is independent of the conventional Hilbert space and path integral approach.

The motion of the electron in the hydrogen atom is called quantum-mechanical Kepler problem. In 1970, McIntosh and Cisneros studied the above-mentioned dynamical system and treated the motion of an electron not only in the Coulomb's potential but also in both magnetic monopole field derived from a vector potential and a centrifugal potential proportional to the square of the pole strength, which is referred later on as the MIC-Kepler problem [7].

Our notation is as follows: $m$ and $k$ are positive constants which denote the mass and the charge of the electron. Moreover Planck's constant $\hbar(>0)$ appears which 
is playing the role of deformation parameter, and $\mu \in \frac{\hbar}{2} \mathbb{Z}$ is the constant specifying the monopole field.

1. We obtain the (Theorem 10) spectral data of the MIC-Kepler problem as follows

$$
\text { Eigenvalues: } \quad E_{n}=\frac{-2 m k^{2}}{\hbar^{2}(n+2)^{2}}, \quad n=0,1,2, \ldots
$$

Dimension of the $(n, l)$ eigenspace: $\quad(n-l+2)(n+l+2) / 4$

where $l \in \mathbb{Z}$ such that $\quad|l| \leq n, l$ and $n$ are simultaneously even or odd.

2. Restricting to the negative energy levels we construct its Green's functions with two local polar coordinates (given in $\$ 5.2$ below). By the transition functions connecting two local trivializations, we show that the local expressions for the Green's function are equivalent, i.e., Green's function is a section of a complex line bundle. (See Theorem 12.)

In 1984, Gracia-Bondía solved the quantum-mechanical Kepler problem in the WWM formalism with the Moyal product. In addition, they showed that the problem was essentially reduced to that of a four-dimensional oscillator with a constraint by means of the Kustaanheimo-Stiefel (KS) transformation in celestial mechanics. They obtained the energy spectrum of bound states and calculated the Green's function for $E>0$ [2].

In 1986, Iwai and Uwano [5] proved that the MIC-Kepler problem is a reduced Hamiltonian system that comes out of the four-dimensional "conformal" Kepler problem, which is closely related to the four-dimensional harmonic oscillator if the associated momentum mapping takes a fixed value $\mu$. Using this formulation in the phase-space, we state that the MIC-Kepler problem can be regarded as the reduced system of the conformal Kepler problem when the momentum mapping of the $S^{1}$ action is set to take a nonzero fixed value $\mu$, and besides that the KS transformation is its principal U(1) bundle $\pi$. In this way, the quantum-mechanical Kepler problem solved by Gracia-Bondía by means of the Moyal product is viewed as the special case when the momentum mapping takes the value zero, i.e., $\mu=0$. In 1988, Iwai and Uwano presented the quantum version by using an operator method and constructed the "quantised" MIC-Kepler problem as a reduction of the "quantised" conformal Kepler problem. A notable theorem is given and the eigenspaces for negative energy are shown concretely. Their dimension and all negative eigenvalues are presented in [6]. Our resultant spectrum coincides with the results of this theorem except that they choose units where $\hbar=1$ and $m$ is set at unity as well $(m=1)$. More important, they differ from us in quantum setting 
as they formulated the quantum system in terms of operators in a Hilbert space, while our system is described in terms of the phase-space common to the classical mechanics. Proir to that Mladenov and Tsanov [8] have studied the MIC-Kepler problem from the view point of geometric quantisation.

Later in 1992, Hoang [3] gave Green's function of the MIC-Kepler problem which is different from ours in quantum formulation as Hoang adopted another one using the method of path integrals. Furthermore, we may emphasize that Green's function obtained in this paper is a kind of section of the vector bundle which is used in Iwai-Uwano [6]. More precisely, we obtain two local expressions and these can be translated into each other through its transition function $g_{-+}$. In our notation, Hoang's result is only a piece of the local expression of the section.

The organization of this paper is as follows. Section 2 presents an outline of phasespace formulation called the WWM formalism. Section 3 is also an outline of previous studies of the MIC-Kepler problem in order to position it as a geometrical problem. In Section 4, we derive the energy spectrum of the MIC-Kepler problem through the Moyal product algebra. In Section 5, we construct the Green's functions of the MIC-Kepler problem on the basis of $*$-exponential function.

\section{2. *-Product on Deformation Quantization}

In [1], it is suggested that "quantization" can be understood as a "deformation" of the algebra $N$ of $\mathbb{C}^{\infty}$ functions on the phase-space with ordinary multiplication of functions. For $f, g \in N$, the new deformed product on $N$ is denoted by $(f, g) \mapsto$ $f * g$. Then we base our calculation on the following product and proposition.

Definition 1. Let $f(\boldsymbol{p}, \boldsymbol{x})$ and $g(\boldsymbol{p}, \boldsymbol{x})$ be two polynomials on the phase space $\left(T^{*} \mathbb{R}^{n}, \mathrm{~d} \boldsymbol{p} \wedge \mathrm{d} \boldsymbol{x}\right)$, where $\mathrm{d} \boldsymbol{p} \wedge \mathrm{d} \boldsymbol{x}=\sum_{j=1}^{n} \mathrm{~d} p_{j} \wedge \mathrm{d} x_{j}$ is the symplectic form. The Moyal product $(f * g)(\boldsymbol{p}, \boldsymbol{x})$ is given by

$$
f * g=f \mathrm{e}^{\frac{\mathrm{i} \hbar}{2} \overleftarrow{\partial \boldsymbol{x}} \wedge \vec{\partial}_{\boldsymbol{p}}} \underset{z}{\leftleftarrows} f \sum_{N=0}^{\infty} \frac{1}{N !}\left(\frac{\mathrm{i} \hbar}{2}\right)^{N}\left(\frac{\overleftarrow{\partial}}{\partial \boldsymbol{x}} \cdot \frac{\vec{\partial}}{\partial \boldsymbol{p}}-\frac{\overleftarrow{\partial}}{\partial \boldsymbol{p}} \cdot \frac{\vec{\partial}}{\partial \boldsymbol{x}}\right)^{N} g
$$

where the partial differentiation operator with superscript $\leftarrow$ operates on $f$ written at the left side of $*$, and the other one with superscript $\rightarrow$ operates on $g$ written at the right side.

Proposition 2. The canonical coordinates $(\boldsymbol{p}, \boldsymbol{x})$ on the classical phase-space $\left(T^{*} \mathbb{R}^{n}, \mathrm{~d} \boldsymbol{p} \wedge \mathrm{d} \boldsymbol{x}\right)$ satisfy the following Canonical Commutation Relations which 
provides generators of the Weyl algebra

$$
\left[p_{j} * p_{k}\right]=0, \quad\left[x_{j} * x_{k}\right]=0, \quad\left[p_{j} * x_{k}\right]=-\delta_{j k} \quad 1 \leq j, k \leq n
$$

where

$$
[f * g] \equiv(f * g-g * f) / \mathrm{i} \hbar \text {. }
$$

For a Hamiltonian function $H(\boldsymbol{x}, \boldsymbol{p})$ on the phase space $\left(T^{*} \mathbb{R}^{n}, \mathrm{~d} \boldsymbol{p} \wedge \mathrm{d} \boldsymbol{x}\right)$ and $t \in \mathbb{R}$ the following series $U_{*}(\boldsymbol{x}, \boldsymbol{p} ; t)$ is called $*$-unitary evolution function, or *-exponential.

$$
\begin{aligned}
U_{*}(\boldsymbol{x}, \boldsymbol{p} ; t) & =\mathrm{e}_{*}^{\frac{\mathrm{i} t}{\hbar} H(\boldsymbol{x p})} \\
& =1+\frac{\mathrm{i} t}{\hbar} H+\frac{1}{2 !}\left(\frac{\mathrm{i} t}{\hbar}\right)^{2} H * H+\cdots+\frac{1}{N !}\left(\frac{\mathrm{i} t}{\hbar}\right)^{N} \overbrace{H * H * \cdots * H}^{N}+\cdots
\end{aligned}
$$

In general, the above power series is not a convergent series. So we consider instead the following differential equation in order to define the $*$-exponential.

$$
-\mathrm{i} \hbar \frac{\partial U_{*}}{\partial t}=H * U_{*}=U_{*} * H, \quad U_{*}(\boldsymbol{x}, \boldsymbol{p} ; 0)=1 \text {. }
$$

We shall use also the notation $\mathrm{e}_{*}^{\frac{\mathrm{i} t}{h} H(\boldsymbol{x}, \boldsymbol{p})}$ which stands for $U_{*}(\boldsymbol{x}, \boldsymbol{p} ; t)$ throughout the paper.

\section{The MIC-Kepler Problem}

\subsection{Classical Theory}

McIntosh and Cisneros [7] studied the dynamical system describing the motion of a charged particle under the influence of Dirac's monopole field and the square inverse centrifugal potential force besides the Coulomb's potential force.

Iwai and Uwano [5] gives the Hamiltonian description for the MIC-Kepler problem as follows.

Theorem 3 (Iwai and Uwano [5], Theorem 3.1). The MIC-Kepler problem is the Hamiltonian system $\left(T^{*} \dot{\mathbb{R}}^{3}, \sigma_{\mu}, H_{\mu}\right)$

$$
\begin{aligned}
H_{\mu}(\boldsymbol{x}, \boldsymbol{p}) & =\frac{1}{2 m}\left(p_{x}^{2}+p_{y}^{2}+p_{z}^{2}\right)+\frac{\mu^{2}}{2 m r^{2}}-\frac{k}{r} \\
\sigma_{\mu} & =\mathrm{d} p_{x} \wedge \mathrm{d} x+\mathrm{d} p_{y} \wedge \mathrm{d} y+\mathrm{d} p_{z} \wedge \mathrm{d} z+\Omega_{\mu}
\end{aligned}
$$


where $\dot{\mathbb{R}}^{3}=\mathbb{R}^{3}-\{0\}, \quad(\boldsymbol{x}, \boldsymbol{p}) \in T^{*} \dot{\mathbb{R}}^{3}, r=\|\boldsymbol{x}\|=\sqrt{x^{2}+y^{2}+z^{2}}$ and $\Omega_{\mu}$ stands for Dirac's monopole field of strength $-\mu$

$$
\Omega_{\mu}=\frac{-\mu}{r^{3}}(x \mathrm{~d} y \wedge \mathrm{d} z+y \mathrm{~d} z \wedge \mathrm{d} x+z \mathrm{~d} x \wedge \mathrm{d} y) .
$$

\subsection{Quantum Theory}

Let us consider the principal $\mathrm{U}(1)$ bundle $\pi: \dot{\mathbb{R}}^{4} \rightarrow \dot{\mathbb{R}}^{3}$ with the free $\mathrm{S}^{1}$-action $\rho$ on $\dot{\mathbb{R}}^{4}$, where the $S^{1}$-bundle $\pi: \dot{\mathbb{R}}^{4} \rightarrow \dot{\mathbb{R}}^{3}$ is contractible to the Hopf fibre bundle $\mathrm{S}^{3} \rightarrow \mathrm{S}^{2}$. For an integer $m$, consider the unitary irreducible representation $\rho_{m}$ of $\mathrm{U}(1) \cong \mathrm{S}^{1}$ on $\mathbb{C}, z \rightarrow \exp (\mathrm{imt} / 2) z, z \in \mathbb{C}$. Let $\mathrm{U}(1)$ act on $\dot{\mathbb{R}}^{4} \times \mathbb{C}$ to the left, then we get the complex line bundle $L_{m}=\left(\dot{\mathbb{R}}^{4} \times_{m} \mathbb{C}, \pi_{m}, \dot{\mathbb{R}}^{3}\right)$, where $\pi_{m}: \dot{\mathbb{R}}^{4} \times_{m} \mathbb{C} \rightarrow$ $\dot{\mathbb{R}}^{3}$ is endowed with the linear connection $\nabla$. The curvature form of $\nabla$ is $\Omega_{m / 2}$, which gives Dirac's monopole field of strength $-m / 2$.

Let $\Gamma_{m}$ be the Hilbert space of square integrable cross sections in $L_{m}$. The quantised MIC-Kepler problem is $\left(\Gamma_{m}, \hat{H}_{m}\right)$ where $\hat{H}_{m}$ is the Hamiltonian operator such that

$$
\hat{H}_{m}=-\frac{1}{2} \sum_{j=1}^{3} \nabla_{j}^{2}+\frac{(m / 2)^{2}}{2 r^{2}}-\frac{k}{r}
$$

and where $\nabla_{j}$ stands for the covariant derivation of $\partial / \partial_{j}$ with respect to the linear connection. Iwai and Uwano showed also that the quantised MIC-Kepler problem $\left(\Gamma_{m}, \hat{H}_{m}\right)$ is obtained by the reduction of the quantised conformal Kepler problem (see Theorem 4.1 in [6]). Using the reduction, Iwai and Uwano obtained the eigenvalues and their multiplicities.

Theorem 4 ([6], Theorem 5.1). The $\rho_{m}$-equivariant eigensubspace $S\left(E_{n} ; m\right)$ for the conformal Kepler problem is in one-to-one correspondence with the eigenspace $q_{m} S\left(E_{n} ; m\right)$ of negative energy $E_{n}=-2 k^{2} /(n+2)^{2}$ for the quantised MIC-Kepler problem $\left(\Gamma_{m}, \hat{H}_{m}\right)$, where $n$ and $m$ are subject to the conditions $|m| \leq n, m$ and $n$ are simultaneously even or odd.

The $q_{m} S\left(E_{n} ; m\right)$ is of dimension $(n-m+2)(n+m+2) / 4$.

\subsection{The MIC-Kepler Problem as Reduced System}

In this subsection, we recall the method of the $S^{1}$-reduction which reduces the conformal Kepler problem on $T^{*} \dot{\mathbb{R}}^{4}$ to the MIC-Kepler problem on $T^{*} \dot{\mathbb{R}}^{3}$.

The $\mathrm{S}^{1}$ action on $\dot{\mathbb{R}}^{4}$ is defined by a $4 \times 4$ matrix $T(\varphi)$

$$
\varphi \in[0,4 \pi], \quad \dot{\mathbb{R}}^{4} \ni \boldsymbol{u} \longmapsto T(\varphi) \boldsymbol{u} \in \dot{\mathbb{R}}^{4}
$$


where $T(\varphi)=\left(\begin{array}{cc}R(\varphi) & O \\ O & R(\varphi)\end{array}\right), \quad R(\varphi)=\left(\begin{array}{cc}\cos \frac{\varphi}{2} & -\sin \frac{\varphi}{2} \\ \sin \frac{\varphi}{2} & \cos \frac{\varphi}{2}\end{array}\right)$

and $\quad \boldsymbol{u}=\left(u_{1}, u_{2}, u_{3}, u_{4}\right)$.

The bundle projection $\pi$ is given as follows

$$
\begin{aligned}
& \pi: \dot{\mathbb{R}}^{4} \longrightarrow \dot{\mathbb{R}}^{3} \\
& \boldsymbol{u} \longmapsto \pi(\boldsymbol{u})=\boldsymbol{x}(\boldsymbol{u}) \quad \text { where }\left\{\begin{array}{l}
x(\boldsymbol{u})=2\left(u_{1} u_{3}+u_{2} u_{4}\right) \\
y(\boldsymbol{u})=2\left(u_{2} u_{3}-u_{1} u_{4}\right) \\
z(\boldsymbol{u})=u_{1}{ }^{2}+u_{2}{ }^{2}-u_{3}{ }^{2}-u_{4}{ }^{2}
\end{array}\right.
\end{aligned}
$$

and we have $u^{2} \equiv u_{1}^{2}+u_{2}^{2}+u_{3}^{2}+u_{4}^{2}=r$.

The $\mathrm{S}^{1}$ action on $T^{*} \dot{\mathbb{R}}^{4}$ is defined by the lift of the one on $\dot{\mathbb{R}}^{4}$ such as in [5], i.e.,

$$
\varphi \in[0,4 \pi], \quad T^{*} \dot{\mathbb{R}}^{4} \ni(\boldsymbol{u}, \boldsymbol{\rho}) \longmapsto(T(\varphi) \boldsymbol{u}, T(\varphi) \boldsymbol{\rho}) \in T^{*} \dot{\mathbb{R}}^{4} .
$$

Let $\psi(\boldsymbol{u}, \boldsymbol{\rho})$ be the momentum mapping of $T^{*} \dot{\mathbb{R}}^{4}$ associated with the above action, i.e., $\psi(\boldsymbol{u}, \boldsymbol{\rho})=\frac{1}{2}\left(-u_{2} \rho_{1}+u_{1} \rho_{2}-u_{4} \rho_{3}+u_{3} \rho_{4}\right)$, given by the defining equation

$$
-\mathrm{d} \psi(\boldsymbol{u}, \boldsymbol{\rho})=\eta\lrcorner \mathrm{d} \theta(\boldsymbol{u}, \boldsymbol{\rho})
$$

$$
=\frac{1}{2}\left(-u_{2}, u_{1},-u_{4}, u_{3},-\rho_{2}, \rho_{1},-\rho_{4}, \rho_{3}\right)-\mathrm{d} \theta
$$

where $\mathrm{d} \theta \equiv \mathrm{d} \boldsymbol{\rho} \wedge \mathrm{d} \boldsymbol{u}=\sum_{j=1}^{4} \mathrm{~d} \rho_{j} \wedge \mathrm{d} u_{j}$.

Next, let $\iota_{\mu}: \psi^{-1}(\mu) \subset T_{\boldsymbol{u}^{*}}^{*} \dot{\mathbb{R}}^{4}$ be the inclusion map. Then the quotient space $\psi^{-1}(\mu) / U(1)$ is diffeomorphic to $T^{*} \dot{\mathbb{R}}^{3}$ and $\pi_{\mu}^{*} \sigma_{\mu}=\iota_{\mu}^{*} \mathrm{~d} \theta$. Hence, we have

Theorem 5 ([5], Theorem 2.5). The reduced phase-space of $\left(T \dot{\mathbb{R}}^{4}, \mathrm{~d} \theta\right)$ is symplectomorphic to $\left(T^{*} \dot{\mathbb{R}}^{3}, \sigma_{\mu}\right)$.

The conformal Kepler problem defined in [5] is the triple $\left(T^{*} \dot{\mathbb{R}}^{4}, \mathrm{~d} \theta, H\right), H(\boldsymbol{u}, \boldsymbol{\rho})$ $=\frac{1}{2 m}\left(\frac{1}{4 u^{2}} \sum_{j=1}^{4} \rho_{j}{ }^{2}\right)-\frac{k}{u^{2}}$. Then we see $\pi_{\mu}^{*} H_{\mu}=\iota_{\mu}^{*} H$ and that the MIC-Kepler problem is obtained by the symplectic reduction of the conformal Kepler problem. (See Theorem 3). 


\section{Solution of Eigenspaces}

\subsection{Harmonic Oscillator}

The harmonic oscillator is deeply related to the conformal Kepler problem. In this subsection we discuss the quantization of the $n$-dimensional harmonic oscillator via the Moyal product. We consider the phase-space $\left(T^{*} \mathbb{R}^{n}, \mathrm{~d} \boldsymbol{p} \wedge \mathrm{d} \boldsymbol{x}\right)$. Let $m$ and $\omega$ be positive constants for the mass of the oscillator and the angular frequency respectively. Let $K(\boldsymbol{x}, \boldsymbol{p})$ denotes the Hamiltonian of the harmonic oscillator defined as follows

$$
K(\boldsymbol{x}, \boldsymbol{p})=\sum_{j=1}^{n} K_{j}\left(x_{j}, p_{j}\right)=\frac{1}{2 m} \sum_{j=1}^{n} p_{j}^{2}+\frac{1}{2} m \omega^{2} \sum_{j=1}^{n} x_{j}^{2}
$$

We consider the following functions for all $j=1, \ldots, n$

$$
\begin{aligned}
a_{j} & =\frac{1}{\sqrt{2}}\left(\sqrt{\frac{m \omega}{\hbar}} x_{j}+\frac{\mathrm{i}}{\sqrt{m \hbar \omega}} p_{j}\right), \quad a_{j}^{+}=\frac{1}{\sqrt{2}}\left(\sqrt{\frac{m \omega}{\hbar}} x_{j}-\frac{\mathrm{i}}{\sqrt{m \hbar \omega}} p_{j}\right) \\
N_{j} & =a_{j}^{+} * a_{j} .
\end{aligned}
$$

The function $a_{j}$ corresponds to the annihilate operator, $a_{j}^{+}$to the create operator and $N_{j}$ to the number operator respectively. Then we get

$$
\begin{aligned}
\boldsymbol{a} \cdot \boldsymbol{a}^{+} & =a_{1} a_{1}^{+}+a_{2} a_{2}^{+}+\cdots+a_{n} a_{n}^{+}=\frac{K(\boldsymbol{x}, \boldsymbol{p})}{\hbar \omega} \\
N_{j} & =a_{j}^{+} a_{j}-\frac{1}{2}, \quad N \equiv N_{1}+N_{2}+\cdots+N_{n}=\boldsymbol{a} \cdot \boldsymbol{a}^{+}-\frac{n}{2} .
\end{aligned}
$$

For all $j=1, \cdots, n$, we introduce

$$
\begin{aligned}
f_{j 0} & =\frac{1}{\pi \hbar} \mathrm{e}^{-2 a_{j}^{+} a_{j}}=\frac{1}{\pi \hbar} \exp \left(-\frac{m \omega}{\hbar} x_{j}^{2}-\frac{1}{m \hbar \omega} p_{j}^{2}\right) \\
f_{k_{j}} & =\frac{1}{k_{j} !} \underbrace{a_{j}^{+} * \cdots * a_{j}^{+}}_{k_{j}} * f_{j 0} * \underbrace{a_{j} * \cdots * a_{j}}_{k_{j}} \\
& =\frac{1}{k_{j} !}\left(a_{j}^{+} *\right)^{k_{j}} f_{j 0}\left(* a_{j}\right)^{k_{j}}, \quad k_{j}=0,1,2 \ldots .
\end{aligned}
$$

We put furthermore

$$
f_{k} \leftleftarrows f_{k_{1}} * f_{k_{2}} * \cdots * f_{k_{n}}, \quad k=0,1,2 \ldots
$$

where $k_{1}, \cdots, k_{n} \in \mathbb{N} \cup\{0\}$ such that $k_{1}+\cdots+k_{n} \equiv k$. 
Then we have the canonical commutation relations

$$
\left[a_{j} * a_{k}\right]=\left[a_{j}^{+} * a_{k}^{+}\right]=0, \quad\left[a_{j} * a_{k}^{+}\right]=-\frac{\mathrm{i}}{\hbar} \delta_{j k}, \quad j, k=1, \cdots, n
$$

which yield the following commutativity

$$
\left[a_{j} * f_{k 0}\right]=\left[a_{j}^{+} * f_{k 0}\right]=0, \quad j \neq k .
$$

Then, we get

$$
\begin{aligned}
N * f_{k} & =k f_{k}, \quad k=0,1,2 \ldots \\
\therefore K * f_{k} & =\hbar \omega\left(\boldsymbol{a} \cdot \boldsymbol{a}^{+}\right) * f_{k}=\hbar \omega\left(N+\frac{n}{2}\right) * f_{k}=\hbar \omega\left(N * f_{k}+\frac{n}{2} * f_{k}\right) \\
& =\hbar \omega\left(k f_{k}+\frac{n}{2} f_{k}\right)=\hbar \omega\left(k+\frac{n}{2}\right) f_{k}
\end{aligned}
$$

furthermore $f_{k} * f_{l}=\frac{1}{(2 \pi \hbar)^{n}} f_{k} \delta_{k l}, \quad k, l=0,1,2, \ldots$

We can get the following proposition.

Proposition 6. The eigenspace of $n$-dimensional harmonic oscillator associated with the eigenvalue $E_{k}=\hbar \omega\left(k+\frac{n}{2}\right), \quad k=0,1,2, \cdots$ is spanned by

$$
\begin{aligned}
f_{k}(\boldsymbol{x}, \boldsymbol{p})=f_{k_{1}} * f_{k_{2}} * \cdots * & f_{k_{n}} \\
& =f_{0}(-1)^{k} L_{k_{1}}\left(4 a_{1}^{+} a_{1}\right) L_{k_{2}}\left(4 a_{2}^{+} a_{2}\right) \cdots L_{k_{n}}\left(4 a_{n}^{+} a_{n}\right)
\end{aligned}
$$

where $k_{1}, \cdots, k_{n} \in \mathbb{N} \cup\{0\}$ such that $k_{1}+\cdots+k_{n} \equiv k$

$$
\begin{aligned}
f_{0} \equiv f_{10} f_{20} \cdots f_{n 0} & =\frac{1}{(\pi \hbar)^{n}} \exp \left(-\frac{m \omega}{\hbar} \sum_{j=1}^{n} x_{j}^{2}-\frac{1}{m \hbar \omega} \sum_{j=1}^{n} p_{j}^{2}\right) \\
L_{k_{j}}\left(4 a_{j}^{+} a_{j}\right) & =\sum_{l=0}^{k_{j}}(-1)^{l} \frac{k_{j} !}{(l !)^{2}\left(k_{j}-l\right) !} \cdot\left(4 a_{j}^{+} a_{j}\right)^{l} \\
4 a_{j}^{+} a_{j} & =4 \frac{K_{j}\left(x_{j}, p_{j}\right)}{\hbar \omega}=2\left(\frac{m \omega}{\hbar} x_{j}^{2}+\frac{1}{m \hbar \omega} p_{j}^{2}\right) .
\end{aligned}
$$

\subsection{The MIC-Kepler Problem}

For a real parameter $E$ let us consider the generalized Hamiltonian $\Phi(\boldsymbol{x}, \boldsymbol{p})$ defined by

$$
\Phi(\boldsymbol{x}, \boldsymbol{p})=r\left(H_{\mu}-E\right) .
$$


Then we have

$$
\left(\pi_{\mu}^{*} \Phi\right)(\boldsymbol{u}, \boldsymbol{\rho})=\frac{1}{8 m}\left(\rho_{1}{ }^{2}+\rho_{2}{ }^{2}+\rho_{3}{ }^{2}+\rho_{4}{ }^{2}\right)-E\left(u_{1}^{2}+u_{2}^{2}+u_{3}^{2}+u_{4}^{2}\right)-k .
$$

The energy hyper surface $E=H_{\mu}$ is equivalent to the condition $\Phi(\boldsymbol{x}, \boldsymbol{p})=0$, which is preserved by the equation of motion. In what follows we consider the case $E<0$. The condition $\left(\pi_{\mu}^{*} \Phi\right)(\boldsymbol{u}, \boldsymbol{\rho})=0$ gives

$$
\frac{1}{2 m} \sum_{j=1}^{4} \rho_{j}^{2}+4|E| \sum_{j=1}^{4} u_{j}^{2}=4 k
$$

and this equation is equivalent to that of four-dimensional harmonic oscillator, if $K(\boldsymbol{u}, \boldsymbol{\rho}) \equiv 4 k$ with $m \omega^{2} / 2 \equiv 4|E|$. Then, by Proposition 6 for the case of fourdimension, we have

$$
E_{n}=\hbar \omega\left(n+\frac{4}{2}\right)=\hbar \omega(n+2) \equiv 4 k, \quad n=0,1,2 \ldots
$$

Then we have $\hbar^{2} \omega^{2}(n+2)^{2}=16 k^{2}$, and from $\omega^{2} \equiv 8|E| / m$, we get

$$
E=\frac{-2 m k^{2}}{\hbar^{2}(n+2)^{2}}, \quad n=0,1,2 \ldots
$$

The conformal Kepler problem introduced by Iwai and Uwano is the triple $\left(T^{*} \dot{\mathbb{R}}^{4}\right.$, $\mathrm{d} \boldsymbol{\rho} \wedge \mathrm{d} \boldsymbol{u}, H)$ such that

$$
H(\boldsymbol{u}, \boldsymbol{\rho})=\frac{1}{2 m}\left(\frac{1}{4 u^{2}} \sum_{j=1}^{4} \rho_{j}^{2}\right)-\frac{k}{u^{2}} .
$$

Note that

$$
\left(\pi_{\mu}^{*} \Phi\right)(\boldsymbol{u}, \boldsymbol{\rho})=u^{2}\left\{\frac{1}{2 m}\left(\frac{1}{4 u^{2}} \sum_{j=1}^{4} \rho_{j}^{2}\right)-\frac{k}{u^{2}}-E\right\}=u^{2}\{H(\boldsymbol{u}, \boldsymbol{\rho})-E\} .
$$

Proposition 6 yields the following one.

Proposition 7. The eigenspace of the conformal Kepler problem associated with the eigenvalue $E_{n}=\frac{-2 m k^{2}}{\hbar^{2}(n+2)^{2}}, \quad n=0,1,2, \ldots$ is spanned by the functions

$$
f_{n}(\boldsymbol{u}, \boldsymbol{\rho})=f_{0}(-1)^{n} L_{n_{1}}\left(4 a_{1}^{+} a_{1}\right) L_{n_{2}}\left(4 a_{2}^{+} a_{2}\right) L_{n_{3}}\left(4 a_{3}^{+} a_{3}\right) L_{n_{4}}\left(4 a_{4}^{+} a_{4}\right)
$$


where $n_{1}, n_{2}, n_{3}, n_{4} \in \mathbb{N} \cup\{0\}$, such that $n_{1}+n_{2}+n_{3}+n_{4} \equiv n$ and for all $j=$ $1,2,3,4$

$$
\begin{gathered}
a_{j} \equiv \frac{1}{\sqrt{2}} \sqrt{\frac{m \omega_{n}}{\hbar}} u_{j}+\frac{\mathrm{i}}{\sqrt{m \hbar \omega_{n}}} \rho_{j}, a_{j}^{+} \equiv \frac{1}{\sqrt{2}} \sqrt{\frac{m \omega_{n}}{\hbar}} u_{j}-\frac{\mathrm{i}}{\sqrt{m \hbar \omega_{n}}} \rho_{j} \\
\hbar \omega_{n} \equiv \frac{4 k}{n+2}, \quad n=0,1,2, \ldots \\
f_{0} \equiv f_{10} f_{20} f_{30} f_{40}=\frac{1}{(\pi \hbar)^{4}} \exp \left(-\frac{m \omega_{n}}{\hbar} \sum_{j=1}^{4} u_{j}^{2}-\frac{1}{m \hbar \omega_{n}} \sum_{j=1}^{4} \rho_{j}^{2}\right) \\
L_{n_{j}}\left(4 a_{j}^{+} a_{j}\right)=\sum_{l=0}^{n_{j}}(-1)^{l} \frac{n_{j} !}{(l !)^{2}\left(n_{j}-l\right) !} \cdot\left(4 a_{j}^{+} a_{j}\right)^{l} .
\end{gathered}
$$

Reduction of conformal Kepler problem by an $\mathrm{S}^{1}$ action is a restriction of the eigenspaces of $H$ to that of $H_{\mu}$, i.e., restriction of the eigenfunctions $f_{n}$ to $\left.f_{n}\right|_{\psi^{-1}(\mu)}$.

Proposition 8. $\left(\left.f\right|_{\psi^{-1}(\mu)}\right)(\boldsymbol{u}, \boldsymbol{\rho})$ satisfies the following $*$-characteristic equation $\psi(\boldsymbol{u}, \boldsymbol{\rho}) * f(\boldsymbol{u}, \boldsymbol{\rho})=\mu * f(\boldsymbol{u}, \boldsymbol{\rho})$.

We need the eigenfunctions which span the eigenspaces of the Hamiltonian and that of the momentum mapping simultaneously. For this purpose, we consider the following functions

$$
\begin{array}{rlrl}
b_{1}^{+}(\boldsymbol{u}, \boldsymbol{\rho}) & =\frac{1}{\sqrt{2}}\left(a_{1}^{+}-\mathrm{i} a_{2}^{+}\right), & b_{1}(\boldsymbol{u}, \boldsymbol{\rho})=\frac{1}{\sqrt{2}}\left(a_{1}+\mathrm{i} a_{2}\right) \\
b_{2}^{+}(\boldsymbol{u}, \boldsymbol{\rho})=\frac{1}{\sqrt{2}}\left(a_{3}^{+}-\mathrm{i} a_{4}^{+}\right), & b_{2}(\boldsymbol{u}, \boldsymbol{\rho})=\frac{1}{\sqrt{2}}\left(a_{3}+\mathrm{i} a_{4}\right) \\
b_{3}^{+}(\boldsymbol{u}, \boldsymbol{\rho})=\frac{1}{\sqrt{2}}\left(a_{1}^{+}+\mathrm{i} a_{2}^{+}\right), & b_{3}(\boldsymbol{u}, \boldsymbol{\rho})=\frac{1}{\sqrt{2}}\left(a_{1}-\mathrm{i} a_{2}\right) \\
b_{4}^{+}(\boldsymbol{u}, \boldsymbol{\rho})=\frac{1}{\sqrt{2}}\left(a_{3}^{+}+\mathrm{i} a_{4}^{+}\right), & b_{4}(\boldsymbol{u}, \boldsymbol{\rho})=\frac{1}{\sqrt{2}}\left(a_{3}-\mathrm{i} a_{4}\right) .
\end{array}
$$

These functions satisfy the following canonical commutation relations

$$
\left[b_{j} * b_{k}\right]=\left[b_{j}^{+} * b_{k}^{+}\right]=0, \quad\left[b_{j} * b_{k}^{+}\right]=-\frac{\mathrm{i}}{\hbar} \delta_{j k}, \quad j, k=1,2,3,4 .
$$

Moreover, we introduce

$$
\begin{array}{ll}
N_{a} \leftleftarrows b_{3}^{+} * b_{3}=b_{3} b_{3}^{+}-\frac{1}{2}, & f_{a 0}=\frac{1}{\pi \hbar} \mathrm{e}^{-2 b_{3}^{+} b_{3}} \\
N_{b}=b_{1}^{+} * b_{1}=b_{1} b_{1}^{+}-\frac{1}{2}, & f_{b 0}=\frac{1}{\pi \hbar} \mathrm{e}^{-2 b_{1}^{+} b_{1}}
\end{array}
$$




$$
\begin{array}{ll}
N_{c} \leftleftarrows b_{2}^{+} * b_{2}=b_{2} b_{2}^{+}-\frac{1}{2}, & f_{c 0}=\frac{1}{\pi \hbar} \mathrm{e}^{-2 b_{2}^{+} b_{2}} \\
N_{d} \leftleftarrows b_{4}^{+} * b_{4}=b_{4} b_{4}^{+}-\frac{1}{2}, & f_{d 0} \leftleftarrows \frac{1}{\pi \hbar} \mathrm{e}^{-2 b_{4}^{+} b_{4}} .
\end{array}
$$

We have

$$
\begin{gathered}
\boldsymbol{b} \cdot \boldsymbol{b}^{+}=\sum_{j=1}^{4} b_{j} b_{j}^{+}=\sum_{j=1}^{4} a_{j} a_{j}^{+}=\boldsymbol{a} \cdot \boldsymbol{a}^{+} \\
\therefore N_{a}+N_{b}+N_{c}+N_{d}=\boldsymbol{b} \cdot \boldsymbol{b}^{+}-2=\boldsymbol{a} \cdot \boldsymbol{a}^{+}-2=N .
\end{gathered}
$$

We also introduce for $n_{a}, n_{b}, n_{c}, n_{d}=0,1,2 \ldots$ the functions

$$
\begin{aligned}
f_{n_{a}} & =\frac{1}{n_{a} !} \underbrace{b_{3}^{+} * \cdots * b_{3}^{+}}_{n_{a}} * f_{a 0} * \underbrace{b_{3} * \cdots * b_{3}}_{n_{a}}=\frac{1}{n_{a} !}\left(b_{3}^{+} *\right)^{n_{a}} f_{a 0}\left(* b_{3}\right)^{n_{a}} \\
f_{n_{b}} & =\frac{1}{n_{b} !} \underbrace{b_{1}^{+} * \cdots * b_{1}^{+}}_{n_{b}} * f_{b 0} * \underbrace{b_{1} * \cdots * b_{1}}_{n_{b}}=\frac{1}{n_{b} !}\left(b_{1}^{+} *\right)^{n_{b}} f_{b 0}\left(* b_{1}\right)^{n_{b}} \\
f_{n_{c}} & =\frac{1}{n_{c} !} \underbrace{b_{2}^{+} * \cdots * b_{2}^{+}}_{n_{c}} * f_{c 0} * \underbrace{b_{2} * \cdots * b_{2}}_{n_{c}}=\frac{1}{n_{c} !}\left(b_{2}^{+} *\right)^{n_{c}} f_{c 0}\left(* b_{2}\right)^{n_{c}} \\
f_{n_{d}} & =\frac{1}{n_{d} !} \underbrace{b_{4}^{+} * \cdots * b_{4}^{+}}_{n_{d}} * f_{d 0} * \underbrace{b_{4} * \cdots * b_{4}}_{n_{d}}=\frac{1}{n_{d} !}\left(b_{4}^{+} *\right)^{n_{d}} f_{d 0}\left(* b_{4}\right)^{n_{d}}
\end{aligned}
$$

and put

$$
f_{n} \leftleftarrows f_{n_{a}} * f_{n_{b}} * f_{n_{c}} * f_{n_{d}}, \quad n=0,1,2 \ldots
$$

where $n_{a}, n_{b}, n_{c}, n_{d} \in \mathbb{N} \cup\{0\}$ such that $n_{a}+n_{b}+n_{c}+n_{d} \equiv n$.

Similarly, we get the following commutation relation

$\left[b_{3} * f_{b 0}\right]=\left[b_{3} * f_{c 0}\right]=\left[b_{3} * f_{d 0}\right]=\left[b_{3}^{+} * f_{b 0}\right]=\left[b_{3}^{+} * f_{c 0}\right]=\left[b_{3}^{+} * f_{d 0}\right]=0$

$\left[b_{1} * f_{a 0}\right]=\left[b_{1} * f_{c 0}\right]=\left[b_{1} * f_{d 0}\right]=\left[b_{1}^{+} * f_{a 0}\right]=\left[b_{1}^{+} * f_{c 0}\right]=\left[b_{1}^{+} * f_{d 0}\right]=0$

$\left[b_{2} * f_{a 0}\right]=\left[b_{2} * f_{b 0}\right]=\left[b_{2} * f_{d 0}\right]=\left[b_{2}^{+} * f_{a 0}\right]=\left[b_{2}^{+} * f_{b 0}\right]=\left[b_{2}^{+} * f_{d 0}\right]=0$

$\left[b_{4} * f_{a 0}\right]=\left[b_{4} * f_{b 0}\right]=\left[b_{4} * f_{c 0}\right]=\left[b_{4}^{+} * f_{a 0}\right]=\left[b_{4}^{+} * f_{b 0}\right]=\left[b_{4}^{+} * f_{c 0}\right]=0$.

In this way we can find that

$$
\left(N_{a}+N_{b}+N_{c}+N_{d}\right) * f_{n}=n f_{n}, \quad \therefore N * f_{n}=n f_{n}
$$

and due to (1)

$$
\left(\boldsymbol{a} \cdot \boldsymbol{a}^{+}-2\right) * f_{n}=n f_{n} \quad \therefore \hbar \omega \boldsymbol{a} \cdot \boldsymbol{a}^{+} * f_{n}=K * f_{n}=\hbar \omega(n+2) f_{n} .
$$


As a result, we get

$$
\begin{array}{ll}
K * f_{n}=\hbar \omega(n+2) f_{n}, & n=0,1,2, \ldots \\
f_{n} * f_{l}=\frac{1}{(2 \pi \hbar)^{4}} f_{n} \delta_{n l}, & n, l=0,1,2, \ldots .
\end{array}
$$

We can reslate the above-mentioned proposition (Proposition 7) as the following.

Proposition 9. The eigenspace of the conformal Kepler problem associated with the eigenvalue $E_{n}=\frac{-2 m k^{2}}{\hbar^{2}(n+2)^{2}}, \quad n=0,1,2, \ldots$ is also spanned $b y$

$$
f_{n}(\boldsymbol{u}, \boldsymbol{\rho})=f_{0}(-1)^{n} L_{n_{a}}\left(4 b_{3}^{+} b_{3}\right) L_{n_{b}}\left(4 b_{1}^{+} b_{1}\right) L_{n_{c}}\left(4 b_{2}^{+} b_{2}\right) L_{n_{d}}\left(4 b_{4}^{+} b_{4}\right)
$$

where $n_{a}, n_{b}, n_{c}, n_{d} \in \mathbb{N} \cup\{0\}$ such that $n_{a}+n_{b}+n_{c}+n_{d} \equiv n$

$$
\begin{aligned}
\hbar \omega_{n} & \equiv \frac{4 k}{n+2}, \quad n=0,1,2, \ldots \\
f_{0} & \equiv f_{a 0} f_{b 0} f_{c 0} f_{d 0}=\frac{1}{(\pi \hbar)^{4}} \exp \left(-\frac{m \omega_{n}}{\hbar} \sum_{j=1}^{4} u_{j}^{2}-\frac{1}{m \hbar \omega_{n}} \sum_{j=1}^{4} \rho_{j}^{2}\right)
\end{aligned}
$$

and for all $(\alpha, j)=(a, 3),(b, 1),(c, 2),(d, 4)$

$$
L_{n_{\alpha}}\left(4 b_{j}^{+} b_{j}\right)=\sum_{l=0}^{n_{\alpha}}(-1)^{l} \frac{n_{\alpha} !}{(l !)^{2}\left(n_{\alpha}-l\right) !} \cdot\left(4 b_{j}^{+} b_{j}\right)^{l} .
$$

In fact,

$$
\begin{aligned}
4 b_{3}^{+} b_{3} & =\frac{m \omega}{\hbar}\left(u_{1}^{2}+u_{2}^{2}\right)+\frac{1}{m \hbar \omega}\left(\rho_{1}^{2}+\rho_{2}{ }^{2}\right)+\frac{2}{\hbar}\left(u_{1} \rho_{2}-u_{2} \rho_{1}\right) \\
4 b_{1}^{+} b_{1} & =\frac{m \omega}{\hbar}\left(u_{1}^{2}+u_{2}^{2}\right)+\frac{1}{m \hbar \omega}\left(\rho_{1}^{2}+\rho_{2}^{2}\right)-\frac{2}{\hbar}\left(u_{1} \rho_{2}-u_{2} \rho_{1}\right) \\
4 b_{2}^{+} b_{2} & =\frac{m \omega}{\hbar}\left(u_{3}^{2}+u_{4}^{2}\right)+\frac{1}{m \hbar \omega}\left(\rho_{3}^{2}+\rho_{4}^{2}\right)-\frac{2}{\hbar}\left(u_{3} \rho_{4}-u_{4} \rho_{3}\right) \\
4 b_{4}^{+} b_{4} & =\frac{m \omega}{\hbar}\left(u_{3}^{2}+u_{4}^{2}\right)+\frac{1}{m \hbar \omega}\left(\rho_{3}^{2}+\rho_{4}^{2}\right)+\frac{2}{\hbar}\left(u_{3} \rho_{4}-u_{4} \rho_{3}\right) .
\end{aligned}
$$

We get

$$
\begin{aligned}
b_{3}^{+} b_{3}-b_{1}^{+} b_{1}-b_{2}^{+} b_{2}+b_{4}^{+} b_{4} & =\frac{1}{\hbar}\left(-u_{2} \rho_{1}+u_{1} \rho_{2}-u_{4} \rho_{3}+u_{3} \rho_{4}\right)=\frac{2}{\hbar} \psi(\boldsymbol{u}, \boldsymbol{\rho}) \\
\therefore \psi(\boldsymbol{u}, \boldsymbol{\rho}) & =\frac{\hbar}{2}\left(b_{3}^{+} b_{3}-b_{1}^{+} b_{1}-b_{2}^{+} b_{2}+b_{4}^{+} b_{4}\right) \\
& =\frac{\hbar}{2}\left(b_{3}^{+} * b_{3}-b_{1}^{+} * b_{1}-b_{2}^{+} * b_{2}+b_{4}^{+} * b_{4}\right) .
\end{aligned}
$$


By (2) and Proposition 8, the conditional equation for reduction is

$$
\left(b_{3}^{+} * b_{3}-b_{1}^{+} * b_{1}-b_{2}^{+} * b_{2}+b_{4}^{+} * b_{4}\right) * f_{n}=\frac{2}{\hbar} \mu * f_{n} .
$$

The left side of (3) can be transformed into the form

$$
\begin{aligned}
\left(b_{3}^{+}\right. & \left.* b_{3}-b_{1}^{+} * b_{1}-b_{2}^{+} * b_{2}+b_{4}^{+} * b_{4}\right) * f_{n_{a}} * f_{n_{b}} * f_{n_{c}} * f_{n_{d}} \\
& =\left(n_{a}-n_{b}-n_{c}+n_{d}\right) f_{n_{a}} * f_{n_{b}} * f_{n_{c}} * f_{n_{d}} \\
& =\left(n_{a}-n_{b}-n_{c}+n_{d}\right) f_{n} .
\end{aligned}
$$

In this way we find the relation

$$
\frac{2}{\hbar} \mu=n_{a}-n_{b}-n_{c}+n_{d} \equiv l, \quad l \in \mathbb{Z} .
$$

Therefore, we get as well

$$
\left\{\begin{array} { l } 
{ \frac { 2 } { \hbar } \mu = l } \\
{ 2 ( n _ { a } + n _ { d } ) = n + l } \\
{ 2 ( n _ { b } + n _ { c } ) = n - l }
\end{array} \quad \therefore \left\{\begin{array}{l}
\mu=\frac{l}{2} \hbar \quad(l \in \mathbb{Z}) \\
|l| \leq n \\
n \text { and } l \text { are simultaneously even or odd. }
\end{array}\right.\right.
$$

Finally, we obtain the theorem.

Theorem 10. The eigenspace of the MIC-Kepler problem associated with the eigenvalue $E_{n}=\frac{-2 m k^{2}}{\hbar^{2}(n+2)^{2}}, \quad n=0,1,2, \ldots$ is spanned $b y$ the functions

$$
f_{n}(\boldsymbol{u}, \boldsymbol{\rho})=f_{0}(-1)^{n} L_{n_{a}}\left(4 b_{3}^{+} b_{3}\right) L_{n_{b}}\left(4 b_{1}^{+} b_{1}\right) L_{n_{c}}\left(4 b_{2}^{+} b_{2}\right) L_{n_{d}}\left(4 b_{4}^{+} b_{4}\right)
$$

where $\quad n_{a}, n_{b}, n_{c}, n_{d} \in \mathbb{N} \cup\{0\}, l \in \mathbb{Z} \quad$ are such that

$$
\left\{\begin{array} { l } 
{ 2 ( n _ { a } + n _ { d } ) \equiv n + l } \\
{ 2 ( n _ { b } + n _ { c } ) \equiv n - l }
\end{array} \quad \text { i.e., } \quad \left\{\begin{array}{l}
|l| \leq n \\
n \text { and l are simultaneously even or odd. }
\end{array}\right.\right.
$$

Its dimension is

$$
\left(\frac{n+l}{2}+1\right)\left(\frac{n-l}{2}+1\right)=\frac{(n+l+2)(n-l+2)}{4} .
$$




\section{Green's Functions}

\subsection{Harmonic Oscillator}

In order to obtain the $*$-exponential function $\mathrm{e}_{*}^{\frac{\mathrm{i} t}{h} K}$ of $n$-dimensional harmonic oscillator, we consider the following differential equation

$$
\begin{array}{rl}
-\mathrm{i} \hbar \frac{\partial}{\partial t} \mathrm{e}_{*}^{\frac{\mathrm{i} t}{h} K}=K * \mathrm{e}_{*}^{\frac{\mathrm{i} t}{\hbar} K}=\mathrm{e}_{*}^{\frac{\mathrm{i} t}{\hbar} K} & * K \\
& =\left(K-\frac{\hbar^{2} \omega^{2}}{4} n \frac{\partial}{\partial K}-\frac{\hbar^{2} \omega^{2}}{4} K \frac{\partial^{2}}{\partial K^{2}}\right) \mathrm{e}_{*}^{\frac{\mathrm{i} t}{h} K}
\end{array}
$$

with the initial condition $\left.\mathrm{e}_{*}^{\frac{\mathrm{i} t}{\hbar} K}\right|_{t=0}=1$. We solve this differential equation explicitly and state

Proposition 11. The *-exponential of n-dimensional harmonic oscillator is given as

$$
\mathrm{e}_{*}^{\frac{\mathrm{i} t}{\hbar} K}=\left(\cos \frac{\omega t}{2}\right)^{-n} \exp \left(\mathrm{i} \frac{2 K}{\hbar \omega} \tan \frac{\omega t}{2}\right), \quad \frac{\omega t}{2} \neq\left(l+\frac{1}{2}\right) \pi, \quad l \in \mathbb{Z} .
$$

Since this $*$-exponential function $\mathrm{e}_{*}^{\frac{\mathrm{i} t}{\hbar} K}$ has singularities on real axis $t(t \geq 0)$, there is a posibility to shift from variable $t$ to $z^{\prime} \equiv t+\mathrm{i} y^{\prime}\left(y^{\prime} \neq 0\right)$ [9].

Then we get

$$
\begin{aligned}
-\mathrm{i} \hbar \frac{\partial}{\partial z^{\prime}} \mathrm{e}_{*}^{\frac{\mathrm{i} z^{\prime}}{h} K} & =K * \mathrm{e}_{*}^{\frac{\mathrm{i} z^{\prime}}{h} K}=\mathrm{e}_{*}^{\frac{\mathrm{i} z^{\prime}}{h} K} * K \\
\mathrm{e}_{*}^{\frac{\mathrm{i} z^{\prime}}{h} K} & =\left(\cos \frac{\omega z^{\prime}}{2}\right)^{-n} \exp \left(\mathrm{i} \frac{2 K}{\hbar \omega} \tan \frac{\omega z^{\prime}}{2}\right) .
\end{aligned}
$$

Let $n=4$, then

$$
K(\boldsymbol{u}, \boldsymbol{\rho})=\frac{1}{2 m} \sum_{j=1}^{4} \rho_{j}^{2}+\frac{1}{2} m \omega^{2} \sum_{j=1}^{4} u_{j}^{2} \equiv \frac{1}{2 m} \rho^{2}+\frac{1}{2} m \omega^{2} u^{2} .
$$

When $y^{\prime}>0$, we can calculate the inverse Fourier-transform of the following * exponential

$$
\mathrm{e}_{*}^{\frac{\mathrm{i} z^{\prime}}{h} K\left(\frac{\boldsymbol{u}_{i}+\boldsymbol{u}_{f}}{2}, \boldsymbol{\rho}\right)}=\left(\cos \frac{\omega z^{\prime}}{2}\right)^{-4} \exp \left\{\mathrm{i} \frac{2}{\hbar \omega} K\left(\frac{\boldsymbol{u}_{i}+\boldsymbol{u}_{f}}{2}, \boldsymbol{\rho}\right) \tan \frac{\omega z^{\prime}}{2}\right\}
$$




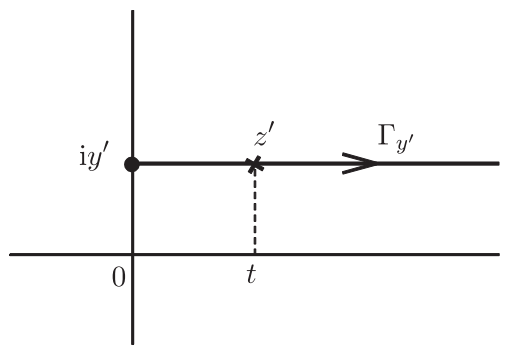

Figure 1. The path of integration $\Gamma_{y^{\prime}}$ for the Laplace transformation of $\tilde{\mathrm{K}}\left(\boldsymbol{u}_{f}, \boldsymbol{u}_{i} ; z^{\prime}\right)$.

where $\boldsymbol{u}_{i}$ and $\boldsymbol{u}_{f}$ denote initial point and final point respectively.

$$
\begin{aligned}
& \frac{1}{(2 \pi \hbar)^{4}} \underbrace{\int_{-\infty}^{\infty} \cdots \int_{-\infty}^{\infty}}_{4}\left(\cos \frac{\omega z^{\prime}}{2}\right)^{-4} \mathrm{e}^{\mathrm{i} \frac{2}{\hbar \omega} K\left(\frac{\boldsymbol{u}_{i}+\boldsymbol{u}_{f}}{2}, \boldsymbol{\rho}\right) \tan \frac{\omega z^{\prime}}{2}} \mathrm{e}^{\frac{\mathrm{i}}{\hbar} \boldsymbol{\rho} \cdot\left(\boldsymbol{u}_{i}-\boldsymbol{u}_{f}\right)} \mathrm{d} \boldsymbol{\rho} \\
= & \frac{-m^{2} \omega^{2}}{4 \pi^{2} \hbar^{2}} \frac{1}{\sin ^{2}\left(\omega z^{\prime}\right)} \exp \left[-\mathrm{i} \frac{m \omega}{2 \hbar} \frac{1}{\sin \left(\omega z^{\prime}\right)}\left\{\left(u_{i}^{2}+u_{f}^{2}\right) \cos \left(\omega z^{\prime}\right)-2 \boldsymbol{u}_{i} \cdot \boldsymbol{u}_{f}\right\}\right] \\
= & \tilde{\mathrm{K}}\left(\boldsymbol{u}_{f}, \boldsymbol{u}_{i} ; z^{\prime}\right)
\end{aligned}
$$

Then we calculate its Green's function by the Laplace transform of (4) as follows.

$$
\begin{aligned}
& \lim _{\operatorname{Im} z^{\prime} \rightarrow+0} \frac{\mathrm{i}}{\hbar} \int_{\Gamma_{y^{\prime}}} \tilde{\mathrm{K}}\left(\boldsymbol{u}_{f}, \boldsymbol{u}_{i} ; z^{\prime}\right) \mathrm{e}^{-\frac{\mathrm{i}}{\hbar}\left(\epsilon-\mathrm{i} y^{\prime}\right) z^{\prime}} \mathrm{d} z^{\prime} \\
= & \lim _{y^{\prime} \rightarrow+0} \frac{\mathrm{i}}{\hbar} \int_{0}^{\infty} \tilde{\mathrm{K}}\left(\boldsymbol{u}_{f}, \boldsymbol{u}_{i} ; t+\mathrm{i} y^{\prime}\right) \mathrm{e}^{-\frac{y^{\prime}+\mathrm{i} \epsilon}{\hbar}\left(t+\mathrm{i} y^{\prime}\right)} \mathrm{d} t \\
= & \frac{-\mathrm{i} m^{2} \omega^{2}}{4 \pi^{2} \hbar^{3}} \lim _{y^{\prime} \rightarrow+0} \int_{0}^{\infty} \mathrm{e}^{-\frac{\mathrm{i}}{\hbar}\left(\epsilon-\mathrm{i} y^{\prime}\right)\left(t+\mathrm{i} y^{\prime}\right)}\left\{\sin \left(\omega t+\mathrm{i} \omega y^{\prime}\right)\right\}^{-2} \\
& \times \exp \left[-\mathrm{i} \frac{m \omega}{2 \hbar} \frac{1}{\sin \left(\omega t+\mathrm{i} \omega y^{\prime}\right)}\left\{\left(u_{i}^{2}+u_{f}^{2}\right) \cos \left(\omega t+\mathrm{i} \omega y^{\prime}\right)-2 \boldsymbol{u}_{i} \cdot \boldsymbol{u}_{f}\right\}\right] \mathrm{d} t \\
= & \mathrm{G}\left(\boldsymbol{u}_{f}, \boldsymbol{u}_{i} ; \epsilon\right) .
\end{aligned}
$$

\subsection{The MIC-Kepler Problem}

We reduce the Green's function of the four-dimensional harmonic oscillator $(\epsilon \equiv 4 k$ and $m \omega^{2} \equiv 8|E|$, i.e., the conformal Kepler problem) to that of the MIC-Kepler 


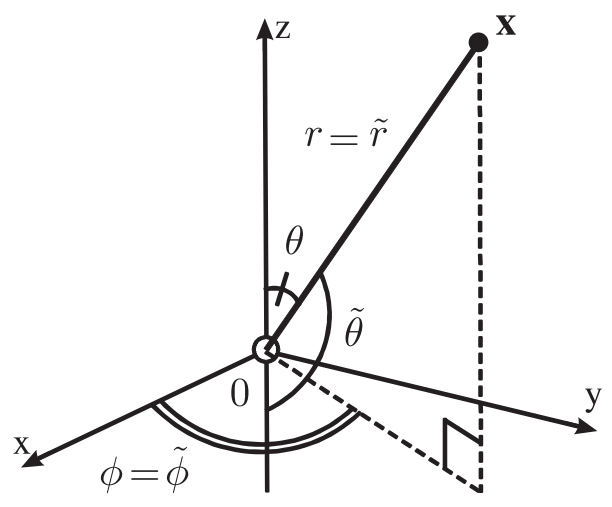

Figure 2. The configuration space $\dot{\mathbb{R}}^{3}=\mathbb{R}^{3}-\{0\}$.

problem by the $S^{1}$ action. We consider the open subsets of $\dot{\mathbb{R}}^{3}=\mathbb{R}^{3}-\{0\}$ such that

$$
\begin{aligned}
& U_{+}=\left\{\mathrm{x}(r, \theta, \phi) \in \dot{\mathbb{R}}^{3} ; r>0,0 \leq \theta<\pi, 0 \leq \phi \leq 2 \pi\right\} \\
& U_{-}=\left\{\mathrm{x}(\tilde{r}, \tilde{\theta}, \tilde{\phi}) \in \dot{\mathbb{R}}^{3} ; \tilde{r}>0,0 \leq \tilde{\theta}<\pi, 0 \leq \tilde{\phi} \leq 2 \pi\right\} .
\end{aligned}
$$

We define two kinds of local coordinate as follows.

$$
\begin{aligned}
& \quad \pi: \pi^{-1}\left(U_{+}\right) \ni u(r, \theta, \phi, \varphi) \longmapsto \mathrm{x}(r, \theta, \phi) \in U_{+} \\
& \begin{array}{l}
x=r \sin \theta \cos \phi \\
y
\end{array}=r \sin \theta \sin \phi \\
& z=r \cos \theta
\end{aligned}\left\{\begin{array}{l}
u_{1}=\sqrt{r} \cos \frac{\theta}{2} \cos \frac{\varphi+\phi}{2}, u_{2}=\sqrt{r} \cos \frac{\theta}{2} \sin \frac{\varphi+\phi}{2} \\
u_{3}=\sqrt{r} \sin \frac{\theta}{2} \cos \frac{\varphi-\phi}{2}, u_{4}=\sqrt{r} \sin \frac{\theta}{2} \sin \frac{\varphi-\phi}{2}
\end{array}\right.
$$

where $r>0,0 \leq \theta<\pi, 0 \leq \phi \leq 2 \pi, 0 \leq \varphi \leq 4 \pi$, and

$$
\begin{gathered}
\pi: \pi^{-1}\left(U_{-}\right) \ni \mathrm{u}(\tilde{r}, \tilde{\theta}, \tilde{\phi}, \tilde{\varphi}) \longmapsto \mathrm{x}(\tilde{r}, \tilde{\theta}, \tilde{\phi}) \in U_{-} \\
\begin{array}{l}
x=\tilde{r} \sin \tilde{\theta} \cos \tilde{\phi} \\
y=\tilde{r} \sin \tilde{\theta} \sin \tilde{\phi} \\
z=-\tilde{r} \cos \tilde{\theta}
\end{array}\left\{\begin{array}{l}
u_{1}=\sqrt{\tilde{r}} \sin \frac{\tilde{\theta}}{2} \cos \frac{\tilde{\varphi}+\tilde{\phi}}{2}, u_{2}=\sqrt{\tilde{r}} \sin \frac{\tilde{\theta}}{2} \sin \frac{\tilde{\varphi}+\tilde{\phi}}{2} \\
u_{3}=\sqrt{\tilde{r}} \cos \frac{\tilde{\theta}}{2} \cos \frac{\tilde{\varphi}-\tilde{\phi}}{2}, u_{4}=\sqrt{\tilde{r}} \cos \frac{\tilde{\theta}}{2} \sin \frac{\tilde{\varphi}-\tilde{\phi}}{2}
\end{array}\right.
\end{gathered}
$$

where $\tilde{r}>0, \quad 0 \leq \tilde{\theta}<\pi, \quad 0 \leq \tilde{\phi} \leq 2 \pi, \quad 2 \pi \leq \tilde{\varphi} \leq 6 \pi$.

Then we have local trivializations $\tau_{ \pm}: \pi^{-1}\left(U_{ \pm}\right) \simeq U_{ \pm} \times S^{1}$, respectively. The transition function

$$
g_{-+}=\tau_{-} \circ \tau_{+}^{-1}: U_{+} \cap U_{-} \times \mathrm{S}^{1} \longrightarrow U_{+} \cap U_{-} \times \mathrm{S}^{1}
$$


is given explicitly as

$$
\begin{aligned}
\boldsymbol{u}(\boldsymbol{x}, \varphi)=\boldsymbol{u}(r, \theta, \phi, \varphi) \mapsto g_{-+}(\boldsymbol{u})(\boldsymbol{x}, \tilde{\varphi}) & =g_{-+}(\boldsymbol{u})(\tilde{r}, \tilde{\theta}, \tilde{\phi}, \tilde{\varphi}) \\
& =g_{-+}(\boldsymbol{u})(r, \pi-\theta, \phi, \varphi+2 \pi)
\end{aligned}
$$

Let $\omega>0$ such that $\omega \neq \omega_{n}=\frac{4 k}{\hbar(n+2)}, \quad n=0,1,2, \ldots$ We calculate the Green's functions of MIC-Kepler problem as follows, where $J_{l}(\xi)$ is the Bessel function.

Theorem 12. i) When $\boldsymbol{u}_{i}, \boldsymbol{u}_{f} \in \pi^{-1}\left(U_{+}\right)$, the Green's function is

$$
\begin{aligned}
\mathrm{G}_{+} & \left(\boldsymbol{r}_{f}, \boldsymbol{r}_{i} ; E=-m \omega^{2} / 8\right) \\
= & r_{f} \int_{0}^{4 \pi} \mathrm{G}\left(\boldsymbol{u}_{f}, \boldsymbol{u}_{i} ; 4 k\right) \exp \left(\mathrm{i} l \frac{\varphi_{i}-\varphi_{f}}{2}\right) \mathrm{d} \varphi_{i} \\
= & (-1)^{\frac{\mu}{\hbar}} \frac{-\mathrm{i} m^{2} \omega^{2}}{16 \pi \hbar^{3}} \lim _{y^{\prime} \rightarrow+0} \int_{0}^{\infty} \mathrm{e}^{-\frac{\mathrm{i}}{\hbar}\left(4 k-\mathrm{i} y^{\prime}\right)\left(t+\mathrm{i} y^{\prime}\right)}\left\{\sin \left(\omega t+\mathrm{i} \omega y^{\prime}\right)\right\}^{-2} \\
& \times \exp \left[-\mathrm{i} \frac{m \omega}{2 \hbar}\left(r_{i}+r_{f}\right) \cot \left(\omega t+\mathrm{i} \omega y^{\prime}\right)-\mathrm{i} \frac{2 \mu}{\hbar} \cdot \frac{\Theta}{2}\right] \\
& \times J_{\frac{2 \mu}{\hbar}}\left(\frac{m \omega}{2 \hbar} \sqrt{2 \boldsymbol{x}_{i} \cdot \boldsymbol{x}_{f}+2 r_{i} r_{f}} \operatorname{cosec}\left(\omega t+\mathrm{i} \omega y^{\prime}\right)\right) \mathrm{d} t
\end{aligned}
$$

where $l=\frac{2 \mu}{\hbar} \in \mathbb{Z}$ and

$$
\frac{\Theta}{2} \equiv \tan ^{-1}\left[\frac{x_{i} y_{f}-y_{i} x_{f}}{r_{i} z_{f}+r_{f} z_{i}} \cdot \frac{z_{i} z_{f}-\sqrt{\left(r_{i}^{2}-z_{i}^{2}\right)\left(r_{f}^{2}-z_{f}^{2}\right)}+r_{i} r_{f}}{z_{i} z_{f}-\sqrt{\left(r_{i}^{2}-z_{i}^{2}\right)\left(r_{f}^{2}-z_{f}^{2}\right)}-\boldsymbol{x}_{i} \cdot \boldsymbol{x}_{f}}\right]
$$

ii) When $\boldsymbol{u}_{i}, \boldsymbol{u}_{f} \in \pi^{-1}\left(U_{-}\right)$, then the Green's function is written as

$$
\begin{aligned}
\mathrm{G}_{-}\left(\tilde{\boldsymbol{r}}_{f}, \tilde{\boldsymbol{r}}_{i} ; E=-m \omega^{2} / 8\right) \\
=\tilde{r}_{f} \int_{2 \pi}^{6 \pi} \mathrm{G}\left(\boldsymbol{u}_{f}, \boldsymbol{u}_{i} ; 4 k\right) \exp \left(\mathrm{i} l \frac{\tilde{\varphi}_{i}-\tilde{\varphi}_{f}}{2}\right) \mathrm{d} \tilde{\varphi}_{i} \\
=(-1)^{\frac{\mu}{\hbar}} \frac{-\mathrm{i} m^{2} \omega^{2}}{16 \pi \hbar^{3}} \lim _{y^{\prime} \rightarrow+0} \int_{0}^{\infty} \mathrm{e}^{-\frac{\mathrm{i}}{\hbar}\left(4 k-\mathrm{i} y^{\prime}\right)\left(t+\mathrm{i} y^{\prime}\right)}\left\{\sin \left(\omega t+\mathrm{i} \omega y^{\prime}\right)\right\}^{-2} \\
\quad \times \exp \left[-\mathrm{i} \frac{m \omega}{2 \hbar}\left(\tilde{r}_{i}+\tilde{r}_{f}\right) \cot \left(\omega t+\mathrm{i} \omega y^{\prime}\right)+\mathrm{i} \frac{2 \mu}{\hbar} \cdot \frac{\tilde{\Theta}}{2}\right] \\
\quad \times J_{\frac{2 \mu}{\hbar}}\left(\frac{m \omega}{2 \hbar} \sqrt{2 \boldsymbol{x}_{i} \cdot \boldsymbol{x}_{f}+2 \tilde{r}_{i} \tilde{r}_{f}} \operatorname{cosec}\left(\omega t+\mathrm{i} \omega y^{\prime}\right)\right) \mathrm{d} t
\end{aligned}
$$


where $l=\frac{2 \mu}{\hbar} \in \mathbb{Z}$ and

$$
\frac{\tilde{\Theta}}{2} \equiv \tan ^{-1}\left[\frac{y_{i} x_{f}-x_{i} y_{f}}{\tilde{r}_{i} z_{f}+\tilde{r}_{f} z_{i}} \cdot \frac{z_{i} z_{f}-\sqrt{\left(\tilde{r}_{i}^{2}-z_{i}^{2}\right)\left(\tilde{r}_{f}^{2}-z_{f}^{2}\right)}+\tilde{r}_{i} \tilde{r}_{f}}{z_{i} z_{f}-\sqrt{\left(\tilde{r}_{i}^{2}-z_{i}^{2}\right)\left(\tilde{r}_{f}^{2}-z_{f}^{2}\right)}-\boldsymbol{x}_{i} \cdot \boldsymbol{x}_{f}}\right]
$$

iii) When $\boldsymbol{u}_{i}, \boldsymbol{u}_{f} \in\left(\pi^{-1}\left(U_{+}\right) \cap \pi^{-1}\left(U_{-}\right)\right)$, and using $g_{-+}$we can easily find

$$
\tan \frac{\tilde{\Theta}}{2}=-\tan \frac{\Theta}{2} \Rightarrow \tilde{\Theta}=-\Theta
$$

which shows that (6) and (7) are equivalent and we can state that the Green's function is a kind of a section.

\section{References}

[1] Bayen F., Flato M., Fronsdal C., Lichnerowicz A. and Sternheimer D., Deformation Theory and Quantization II. Physical Applications, Ann. Phys. 110 (1978) 111-151.

[2] Gracia-Bondía J., Hydrogen Atom in the Phase-Space Formulation of Quantum Mechanics, Phys. Rev. A 30 (1984) 691-697.

[3] Hoang L., On the Green Function for the MIC-Kepler Problem, Izv. Akad. Nauk Belarusi, Ser. Fiz. \& Mat. Nauk 2 (1992) 76-80.

[4] Hoang L., Komarov L. and Romanova T., On the Coulomb Green Function, J. Phys. A: Math. \& Gen. 22 (1989) 1543-1552.

[5] Iwai T. and Uwano Y., The Four-dimensional Conformal Kepler Problem Reduces to the Three-dimensional Kepler Problem with a Centrifugal Potential and Dirac's Monopole Field. Classical Theory, J. Math. Phys. 27 (1986) 1523-1529.

[6] Iwai T. and Uwano Y., The Quantised MIC-Kepler Problem and Its Symmetry Group for Negative Energies, J. Phys. A: Math. \& Gen. 21 (1988) 4083-4104.

[7] McIntosh H. and Cisneros A., Degeneracy in the Presence of a Magnetic Monopole, J. Math. Phys. 11 (1970) 896-916.

[8] Mladenov I. and Tsanov V., Geometric Quantisation of the MIC-Kepler Problem, J. Phys. A: Math. \& Gen. 20 (1987) 5865-5871. 
[9] Moreno C. and Silva J., Star-Products, Spectral Analysis, and Hyperfunctions, In: Conférence Moshé Flato 1999, vol. 2, G. Dito and D. Sternheimer (Eds), Kluwer Academic Publishers, Amsterdam 2000, pp 211-224.

[10] Zachos C., Fairlie D. and Curtright T., Quantum Mechanics in Phase Space, World Scientific, Singapore 2005.

Tomoyo Kanazawa

Akira Yoshioka

Department of Mathematics

Tokyo University of Science

Kagurazaka1-3, Shinjuku-ku

Tokyo 162-8601, JAPAN

E-mail address: tomo-n-jewel@qit.ne.jp

E-mail address: yoshioka@rs.kagu.tus.ac.jp 